\title{
L’adoption du semis direct sous couvert végétal : transition douce ou rupture?
}

\author{
Damien Derrouch*, Fabrice Dessaint, Émeline Felten et Bruno Chauvel \\ Agroécologie, AgroSup Dijon, INRAE, Univ. Bourgogne, Univ. Bourgogne Franche-Comté, F-21000 Dijon, France
}

\begin{abstract}
Résumé - Le semis direct sous couvert repose sur l'application simultanée et continue de trois principes: la réduction quasi-totale du travail du sol, une couverture organique des sols et une rotation diversifiée. Ce système agricole adopté en France à partir des années 2000 sous l'impulsion de groupes d'agriculteurs est en extension. Pour les agriculteurs, adopter un nouveau système agricole revient à modifier, de manière plus ou moins importante, leurs pratiques. Concernant le semis direct sous couvert végétal, peu d'informations existent. Cet article a pour objectif d'éclairer les modifications que peut induire sa mise en place. Pour cela, il s'appuie sur les réponses de 425 agriculteurs français à une enquête en ligne. Pour $30 \%$ des agriculteurs, le semis direct sous couvert constitue une modification complète du système agricole. La réduction quasitotale du travail du sol est le principe qui entraîne le plus de modifications (pour $96 \%$ des agriculteurs). Au contraire, la diversification de la rotation connaît le moins de modifications (48\% des agriculteurs). L'absence d'une modification de la rotation peut s'expliquer par une rotation déjà diversifiée en place ou l'incapacité pour les agriculteurs d'ajouter de nouvelles cultures à leur rotation. L'optimisation de la couverture végétale du sol entraîne une modification des pratiques pour $67 \%$ des agriculteurs. Durant les premières années, les agriculteurs s'orientent majoritairement vers l'utilisation de couverts temporaires plurispécifiques. Bouleversant certains fondamentaux de l'agriculture, ces agriculteurs minimisent les risques encourus en favorisant une transition progressive et en partageant les connaissances acquises.
\end{abstract}

Mots clés : enquête / agriculture de conservation / non-travail du sol / couverture du sol / diversification des cultures

\begin{abstract}
Adoption of direct seeding under cover: soft transition or rupture? Direct seeding under cover meets three principles applied simultaneously and continuously: almost total reduction of tillage, maximum soil coverage and diversification of rotation. Its adoption started in France in the 2000s and today seems to convince more and more farmers. For farmers, the adoption of a new system means changing practices, by important ways or not. For direct seeding under cover, few is known about the required changes. The purpose of this article is to clarify the changes that can occur for farmers when adopting direct seeding under cover. An online survey was conducted: 425 French farmers responded. Results show that the adoption of direct seeding under cover has deeply changed the practices of $30 \%$ of farmers. For others, changes concern only one or two principles. The most important changes (for $96 \%$ of farmers) concern the almost total reduction of soil tillage principle. On the contrary, changes are less important for the diversification of rotation. Only $48 \%$ of farmer modify their crop rotation when adopting direct seeding under cover. Optimizing soil coverage represents a change for $67 \%$ of farmers. During first years, soil coverage is mainly achieved through multi-species temporary cover crops. By upsetting fundamentals of conventional farming, these farmers seems to minimize risks when adopting direct seeding under cover by a soft transition way and by sharing knowledge.
\end{abstract}

Keywords: surveys / conservation agriculture / conservation tillage / land cover / crop diversification

\section{Introduction}

Le semis direct sous couvert (SDSC) est un système agricole combinant l'application simultanée et continue de

\footnotetext{
$\overline{\text { *Auteur de correspondance }}$ : damien.derrouch@inrae.fr
}

trois principes agronomiques: (1) une réduction quasi-totale du travail du sol, (2) une couverture organique des sols, vivante ou morte, temporaire ou permanente et (3) une rotation diversifiée (Fig. 1). À l'étranger, le SDSC correspond à l'agriculture de conservation définie par Reicosky (2015). En France, le SDSC est une déclinaison de l'agriculture de 
conservation dans laquelle le travail du sol se limite à l'ouverture du sillon lors du semis (pratique d'implantation appelée «semis direct»). Adopté en France sous l'impulsion de groupes d'agriculteurs à partir des années 2000, ce système agricole concernait, en 2009, 200000 hectares (Derpsch et al., 2010) et semble convaincre de plus en plus d'agriculteurs. L'approche du SDSC est innovante du fait de l'association de ces trois principes, mais chaque principe peut être individuellement applicable.

La réduction quasi-totale du travail du sol, par le biais du semis direct, représente pour les agriculteurs un avantage économique (diminution du temps de travail et du coût énergétique) (Šarauskis et al., 2014). Cette pratique d'implantation peut également fournir des bénéfices environnementaux: minimiser et prévenir l'érosion du sol (Cameira et al., 2003), améliorer la structure et la porosité du sol (Schwen et al., 2011) et améliorer la vie du sol, en particulier en favorisant les espèces décrites comme bénéfiques (vers de terre, carabes, etc.) (House et Parmelee, 1985). Néanmoins, le semis direct peut aussi favoriser des espèces décrites comme plus problématiques d'un point de vue de leur gestion (adventices, limaces, rongeurs, taupes) (Dorado et LópezFando, 2006).

La couverture végétale du sol concerne aussi bien la période de culture que la période d'interculture, période allant de la récolte d'une culture au semis de la culture suivante. En SDSC, la gamme de couverture du sol utilisable par les agriculteurs est large: couvert temporaire (une ou plusieurs espèces annuelles semées pour une courte durée), couvert permanent (une ou plusieurs espèces pluriannuelles semées pour une durée variable), repousses de cultures (repousses spontanées post-récolte) et résidus de cultures ou de couvert (parties végétales non récoltées et laissées sur le sol). La mise en place d'une couverture végétale d'un sol peut répondre à différents objectifs: protéger le sol de l'érosion (Laloy et Bielders, 2010), fixer l'azote atmosphérique grâce aux légumineuses (Parr et al., 2011), réduire les pertes de nitrates (Constantin et al., 2011), améliorer la porosité du sol (Carof et al., 2007) et limiter la germination et l'émergence de certaines espèces adventices annuelles (Cordeau et al., 2018). Les services fournis par cette couverture végétale restent cependant fortement influencés par sa gestion (espèces choisies, date et densité de semis, méthode de destruction) (Osipitan et al., 2019). Optimiser une couverture végétale nécessite, pour les agriculteurs, une technicité plus importante. Mal gérée, cette couverture peut devenir compétitive vis-à-vis de la culture suivante (Nielsen et al., 2016).

La diversification de la rotation peut tout d'abord représenter un avantage économique et organisationnel (répartition de la charge de travail, sécurisation économique par rapport aux variations des prix du marché et aux aléas climatiques), mais aussi constituer un levier efficace de gestion des maladies, des adventices et des ravageurs. Son action peut être préventive, en rompant leur cycle de vie (Barzman et al., 2015) mais aussi curative, par l'alternance des modes d'action des pesticides utilisés. D'après Reicosky (2015), une rotation diversifiée est composée à minima de trois cultures différentes, dont une légumineuse.

Appliqués simultanément et en continu, les trois principes du SDSC agissent de manière complémentaire afin de remplir les objectifs fixés par les agriculteurs. Possible en Europe depuis les années 1970 (Baeumer et Bakermans, 1974), et applicable sur une grande partie du territoire français (Laurent, 2015), dans une grande diversité de conditions pédoclimatiques, techniques et socioéconomiques, le SDSC a cependant tardé à se développer. Deux causes principales ont limité son adoption: le contrôle difficile des plantes adventices et l'absence de matériel agricole adapté pour le semis. L'arrivée sur le marché de nouvelles molécules non sélectives, dont le glyphosate (voir l'encadré 1 sur l'utilisation du glyphosate en SDSC) et le glufosinate, ainsi que l'importation de semoirs adaptés sur le continent européen, ont levé en partie ces limitations.

Encadré 1. Utilisation actuelle du glyphosate par les agriculteurs en SDSC en France.

Le glyphosate ( $\mathrm{N}$-(phosphonométhyl)glycine, $\mathrm{C}_{3} \mathrm{H}_{8} \mathrm{NO}_{5} \mathrm{P}$ ) est une substance active herbicide non sélective à pénétration foliaire qui est homologuée en France depuis 1974. Absorbé par les organes aériens et capable de migrer jusqu'au système radiculaire, il peut être utilisé pendant la période d'interculture ou entre les rangs des vignes, des arbres fruitiers ou de cultures comme les bananiers. Depuis les retraits du paraquat (2007), de l'aminotriazole (2016) et du glufosinate (2018), le glyphosate, seul ou en association, constitue en France l'une des dernières molécules utilisables pendant l'interculture avec quelques autres molécules (acide pélargonique, dicamba, 2,4-D) à spectres d'actions et à rapport efficacité-coût plus réduits. La dose d'utilisation homologuée en interculture en France pour une concentration de $360 \mathrm{~g} / \mathrm{L}$ est de $3 \mathrm{~L} /$ ha sur les graminées annuelles, $6 \mathrm{~L} /$ ha pour les eudicotylédones et $7 \mathrm{~L} /$ ha pour les plantes vivaces. Son utilisation très fréquente en fait le premier herbicide en France, avec près de 9000 tonnes par an.

Selon les données de notre étude, le glyphosate est utilisé à ce jour (2018-2019), par 97\% des agriculteurs en SDSC. La fréquence d'utilisation varie en fonction des répondants : $90 \%$ l'utilisent tous les ans, $7 \%$ tous les deux à trois ans et $3 \%$ de manière sporadique. Son utilisation répond principalement à trois objectifs : contrôler (1) le couvert végétal $(6 \%)$, (2) la flore adventice (42\% dont $22 \%$ concernant uniquement la gestion des graminées), (3) simultanément le couvert et la flore adventice (50\% des répondants). La dose moyenne annuelle s'échelonne, pour une équivalence de concentration de $360 \mathrm{~g} / \mathrm{L}$, de 0,1 à $0,5 \mathrm{~L} /$ ha $(1 \%$ des agriculteurs) à plus de $3,5 \mathrm{~L} / \mathrm{ha}$ ( $1 \%$ des agriculteurs). La majorité des agriculteurs $(77 \%)$ utilisent une dose de glyphosate qui ne dépasse pas à 1,5 à $2 \mathrm{~L} / \mathrm{ha} / \mathrm{an}$.

Pour les agriculteurs, tout changement de système agricole nécessite d'abandonner, de remplacer, d'élargir et/ou d'acquérir de nouvelles pratiques, ce qui peut représenter une période délicate. Bien que les modifications de certains systèmes agricoles, comme par exemple lors de la conversion à 


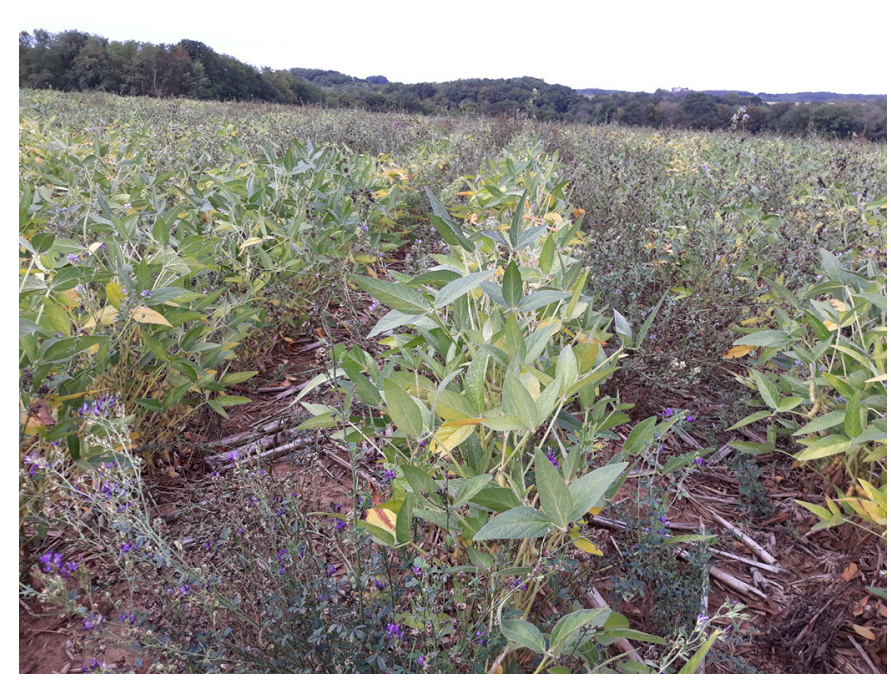

Fig. 1. Culture de soja dans un couvert permanent de luzerne.

Fig. 1. Soybeans with permanent alfalfa cover.

l'agriculture biologique (Schipanski et al., 2014), aient été déjà étudiées, il existe peu d'informations concernant l'adoption du SDSC. L'adoption du SDSC se fait-elle progressivement ou résulte-t-elle d'une modification majeure de l'ensemble des pratiques du système agricole? Durant les premières années en SDSC, les agriculteurs ont-ils tous recours à des pratiques similaires?

Dans cette étude, nous avons choisi de répondre à ces questions en réalisant un état des lieux sur les choix réalisés par les agriculteurs lors de l'adoption du SDSC. Pour être le plus représentatif possible de la réalité, les agriculteurs, acteurs du changement, ont directement été interrogés par le biais d'une enquête.

\section{L'enquête}

L'enquête en ligne est une méthode efficace pour capter les pratiques directement mises en place par les acteurs concernés (Peigné et al., 2016). Elle présente les avantages d'obtenir un grand nombre de réponses à faible coût et de fournir une couverture temporelle et spatiale plus importante que par des méthodes d'entretiens présentiels.

L'enquête utilisée dans cette étude a été créée pour les agriculteurs français en SDSC ou ayant eu une expérience dans ce système agricole, avec un focus sur l'aspect agronomique. Les agriculteurs devaient décrire leurs pratiques de manière représentative de l'ensemble de leurs parcelles en SDSC pour deux périodes : (i) avant l'adoption du SDSC et (ii) durant la ou les premières années en SDSC. Les dix-huit questions utilisées sont présentées en données supplémentaires.

L'outil d'enquête LimeSurvey (https://www.limesurvey. org/fr/) a été utilisé pour réaliser le questionnaire. L'enquête a été mise en ligne du 9 novembre 2018 au 4 mars 2019. Elle a été diffusée via des responsables de réseaux impliqués dans l'agriculture de conservation (Association pour la Promotion d'une Agriculture Durable (APAD), Biodiversité Agriculture Sol et Environnement (BASE), Groupements d'Intérêt Économique et Environnemental (GIEE) Du Sol Eau Soleil,
Association Occitane de Conservation des Sols (AOC Sols), Club AgroEcos, Groupements d'Intérêt Économique et Environnemental Mobilisation des Agriculteurs dans la Gestion et l'Évaluation de systèmes de cultures sous couvert de Légumineuses pour La maîtrise des Adventices et de l'N (GIEE MAGELLAN), Clé de Sol), par les chambres d'agriculture et par le réseau social Facebook. Toutes les informations collectées sont anonymes.

\subsection{Profil des agriculteurs}

Sur un total de 1167 connexions, 425 questionnaires français complets ont été enregistrés. La surface en SDSC échantillonnée s'élève à 49500 hectares, soit $25 \%$ de la surface estimée en SDSC en 2009. L'enquête a été remplie par des agriculteurs provenant de toutes les régions de France : 69 en Bourgogne-Franche-Comté, 62 en Grand Est, 58 en Occitanie, 57 en Centre-Val de Loire, 44 en NouvelleAquitaine, 34 en Auvergne-Rhône-Alpes, 23 en Hauts-deFrance, 22 en Pays de la Loire, 22 en Normandie, 19 en Île-deFrance, 12 en Bretagne et 3 en Provence-Alpes-Côte d'Azur. Le déséquilibre de réponses entre les régions peut résulter d'une différence plus ou moins importante dans le nombre d'agriculteurs en SDSC et/ou de la diffusion du questionnaire via certains réseaux. Le SDSC concerne aussi bien les céréaliers $(55 \%)$ que les polyculteurs-éleveurs $(45 \%)$. De nombreuses conditions pédologiques sont représentées avec une majorité de sols argileux, argilo-limoneux et limonoargileux et une minorité de sols limoneux fins. La première adoption du SDSC date de 1992, mais 50\% des adoptions ont été réalisées après 2015.

\section{Résultats}

Sur les 425 agriculteurs ayant répondu, 126(30\%) ont modifié de manière importante (trois principes concernés) leur système agricole (Fig. 2). Pour les autres agriculteurs (70\%), les modifications ont été moins importantes et n'ont concerné qu'un ou deux principes du SDSC. Le nombre d'agriculteurs qui appliquaient déjà un des principes du SDSC avant son adoption était de 220 (rotation diversifiée pour 146, mise en place de couverts végétaux pour 67 , utilisation du semis direct pour 7 ), soit $52 \%$ de l'ensemble des agriculteurs. Le nombre d'agriculteurs qui appliquaient déjà simultanément deux principes était de 77 (majoritairement la mise en place de couverts végétaux et l'utilisation d'une rotation diversifiée), soit $18 \%$.

La limitation du travail du sol au semis direct, unique technique d'implantation en SDSC, a constitué la modification majeure pour les agriculteurs. Avant l'adoption du SDSC, cette pratique concernait seulement 18 agriculteurs (4\%). Les autres agriculteurs mobilisaient plus ou moins fortement le travail du sol: $275(65 \%)$ étaient en techniques culturales simplifiées (TCS), impliquant de ne pas recourir au labour, et $132(31 \%)$ labouraient le sol. Le labour, action de retournement de l'horizon de surface sur une certaine profondeur, était pratiqué de manière systématique par 51 agriculteurs $(13 \%)$ et de manière occasionnelle par 81 agriculteurs $(18 \%)$.

À l'opposé, la diversification de la rotation est le principe qui a rencontré le moins de modifications. Au total, 


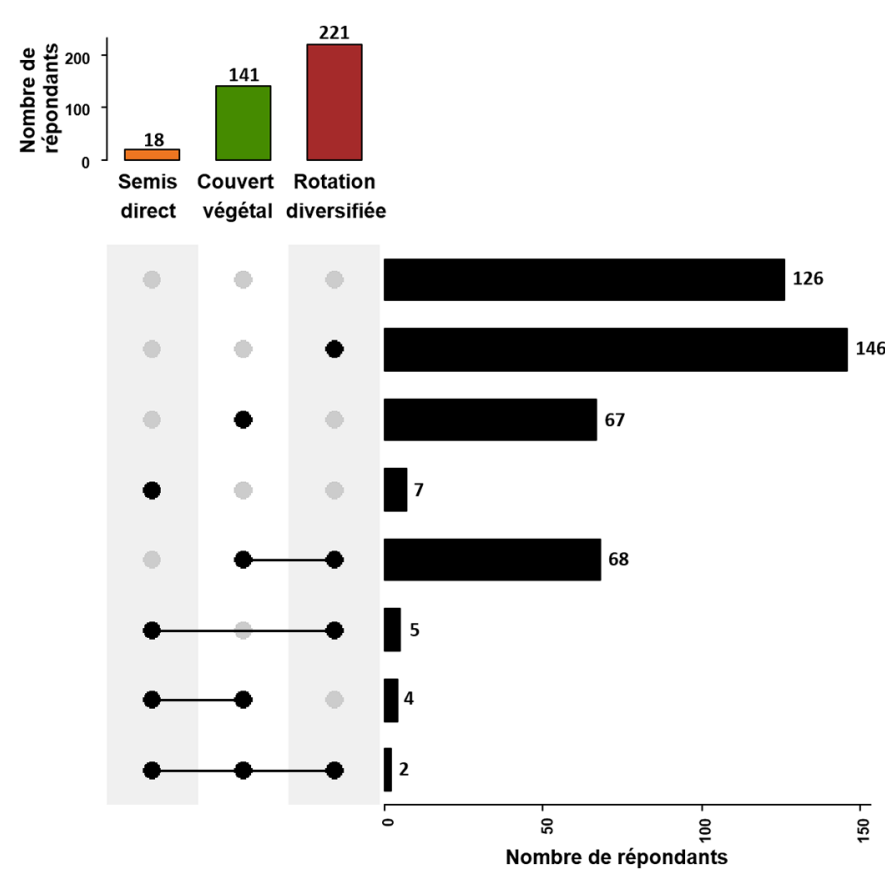

Fig. 2. Utilisation des principes du semis direct sous couvert végétal par les agriculteurs durant la période précédant l'adoption. La réduction quasi-totale du travail du sol est représentée par le semis direct, la couverture végétale du sol par la mise en place d'un couvert végétal et la rotation diversifiée par l'absence de modification de la rotation à l'adoption du SDSC.

Fig. 2. Use of direct seeding under cover principles during the period preceding the adoption of direct seeding under cover. The almost total reduction in tillage is represented by direct seeding, soil coverage by adding a cover crop and diversified rotation by the absence of crop rotation change when adopting direct seeding under cover.

221 agriculteurs soit $52 \%$ du total, n'ont pas modifié leur rotation à l'adoption du SDSC. Pour les 204 agriculteurs (48\%) qui ont modifié leur rotation, plusieurs voies ont été choisies : 60 ont modifié uniquement la fréquence de retour des grandes familles de cultures, 117 ont ajouté/supprimé une grande famille de cultures et 27 ont choisi une rotation non prédéfinie («Autre rotation»). Pour chacune des grandes familles de cultures proposées, les ajouts ou les retraits réalisés par les 117 agriculteurs à l'adoption du SDSC ont été recensés (Tab. 1). Le nombre d'ajouts par rapport au nombre de retraits confirme tout d'abord la diversification de la rotation. Les légumineuses sont ajoutées dans 81 rotations, soit $66 \%$ des ajouts. Concernant les retraits, le choix des agriculteurs s'est tourné majoritairement sur les prairies (41\% des retraits). Durant les premières années en SDSC, les rotations les plus fréquentes comportent à minima le couple céréales à pailles oléagineux $(12 \%)$, auquel s'ajoutent les légumineuses $(17 \%)$ puis le maïs $(22 \%)$. Pour $15 \%$ des répondants, la rotation résulte d'un comportement opportuniste et de l'absence d'une rotation prédéfinie («Autre rotation »).

Le troisième principe, la couverture végétale du sol, était déjà appliqué par 141 agriculteurs (33\%) avant l'adoption du SDSC. Les modifications des pratiques liées à ce principe ont concerné 284 agriculteurs (67\%). Avec l'adoption du SDSC, la gamme d'utilisation de couvertures végétales s'élargit.
Tableau 1. Modifications (ajouts ou retraits) effectuées à l'adoption du semis direct sous couvert végétal par les 117 agriculteurs ayant modifié les grandes familles de culture présentes dans leur rotation. Table 1. Crop rotation changes (additions or withdrawals) effected when adopting direct seeding under cover by the 117 farmers who modified families of cultures in their rotation.

\begin{tabular}{lcc}
\hline Grandes familles & \multicolumn{2}{c}{$\begin{array}{c}\text { Modification de la rotation par les } \\
\text { agriculteurs à l'adoption du SDSC }\end{array}$} \\
\cline { 2 - 3 } & Nombre d'ajouts & Nombre de retraits \\
\hline Céréales à pailles & 5 & 0 \\
Légumineuses & 81 & 5 \\
Maïs & 17 & 7 \\
Oléagineux & 15 & 7 \\
Prairie & 4 & 13 \\
\hline
\end{tabular}

Plusieurs couvertures végétales peuvent être mobilisées simultanément sur l'exploitation en fonction de la rotation, mais le choix des agriculteurs se tourne majoritairement vers les couverts temporaires (386 agriculteurs, soit $91 \%$ ). Hormis certaines exceptions ( $3 \%$ ), ces couverts sont plurispécifiques : $50 \%$ d'entre eux sont composés au minimum par cinq espèces. La féverole (Vicia faba; 53\%), la phacélie (Phacelia tanacetifolia; $46 \%$ ), les vesces (Vicia spp.; $39 \%$ ), le tournesol (Helianthus annuus; $31 \%$ ), les avoines (Avena spp.; $31 \%$ ), les moutardes (Sinapis spp. et Brassica spp.; 26\%), le radis fourrager (Raphanus sativus; $25 \%$ ), le sarrasin (Fagopyrum esculentum; $24 \%$ ) et le pois fourrager (Pisum sativum ; $24 \%$ ) sont les espèces les plus utilisées durant les premières années de SDSC. Durant cette même période, 76 agriculteurs (18\%) utilisent des couverts permanents. Ces couverts sont composés exclusivement de légumineuses: la luzerne cultivée (Medicago sativa; $31 \%$ ), le trèfle blanc (Trifolium repens ; $34 \%$ ), le trèfle violet (Trifolium pratense; $15 \%$ ) et le lotier corniculé (Lotus corniculatus; $13 \%$ ). Enfin, les résidus de cultures et les repousses sont utilisés respectivement par 98 et 100 agriculteurs $(23$ et $24 \%)$.

À l'échelle de l'exploitation, 245 agriculteurs (58\%) ont adopté le SDSC de manière progressive (parcelle par parcelle) et 180 agriculteurs $(42 \%)$ directement sur l'ensemble de l'exploitation. La méthode d'adoption choisie ne conduit pas au même résultat. En se concentrant sur les agriculteurs ayant eu le temps de réaliser une rotation (i.e. adoption avant 2015), seulement $32 \%$ des agriculteurs ayant adopté le SDSC de manière progressive possèdent actuellement l'ensemble de l'exploitation en SDSC. Ce chiffre s'élève à $89 \%$ pour les agriculteurs ayant adopté directement le SDSC sur la totalité de leur exploitation. A l'adoption du SDSC, 172 agriculteurs (40\%) ont choisi de débuter le SDSC par une culture en particulier, les principales étant : le blé d'hiver ( $48 \%)$, le colza $(18 \%)$ et le maïs $(11 \%)$.

\section{Discussion}

La modification principale à l'adoption du SDSC repose sur la réduction quasi-totale du travail du sol (pour $96 \%$ des 
agriculteurs). Généralisé sur l'ensemble des exploitations avec l'industrialisation de l'agriculture, le travail du sol s'est ancré dans les traditions agricoles. Multi-objectifs (préparation du lit de semence, gestion préventive et curative des adventices et des ravageurs, enfouissement des résidus et des effluents d'élevage, etc.), il est devenu la base du raisonnement agricole. Limiter le travail du sol génère donc des contraintes importantes pour les agriculteurs. Bijttebier et al. (2018) en ont identifié plusieurs, dont des contraintes techniques (remise en cause des connaissances et des méthodes, matériel non adapté), sociales (regard des voisins) et économiques (risque d'échec). Pour atténuer un maximum ces contraintes, les agriculteurs ayant répondu à notre enquête ont opté majoritairement $(65 \%)$ pour une réduction progressive du travail du sol (labour puis TCS puis semis direct). Le changement peut être considéré comme plus radical pour les $31 \%$ d'agriculteurs qui sont passés directement du labour au semis direct. Cette transition plus brutale a pu être facilitée par l'existence de connaissances antérieures acquises sur le continent américain et/ ou par le partage actif de retours d'expériences au sein des réseaux d'agriculteurs (Laurent, 2015).

La diversification de la rotation est le principe le moins modifié lors du passage au SDSC. Plus de la moitié (52\%) des agriculteurs n'ont pas modifié leur rotation. La diversification de la rotation est un sujet fortement documenté et conseillé depuis plusieurs décennies par la recherche en vue de résoudre des problèmes comme l'apparition de résistances aux pesticides (Clarke et al., 1997). La diversification des rotations devient même obligatoire dans la réforme de la PAC 2015-2019 qui fixe, entre autres, un nombre de cultures à respecter sur les exploitations. Avant d'adopter le SDSC, la diversification de leur rotation peut donc déjà avoir été réalisée. Une autre origine à cette absence de modification de rotation peut également être envisageable: l'existence de nombreuses contraintes techniques, organisationnelles, économiques et environnementales à la diversification d'une rotation (Meynard et al., 2013). En SDSC, ces contraintes peuvent être accentuées, notamment pour les cultures de printemps/été, du fait des difficultés d'implantation. Concernant les $48 \%$ d'agriculteurs ayant modifié leur rotation, leur choix s'est dirigé majoritairement vers l'ajout de cultures de légumineuses dans la rotation. Outre les effets évoqués précédemment sur les bénéfices d'une rotation diversifiée, l'ajout de légumineuses permet également un enrichissement de la parcelle en azote (Parr et al., 2011).

L'optimisation de la couverture végétale du sol n'a pas entraîné de modifications des pratiques pour $33 \%$ des agriculteurs lors du passage au SDSC. Comme la diversification de la rotation, ce principe peut être applicable dans des systèmes agricoles avec travail du sol. Sa mise en place est, suite à la Directive n ${ }^{\circ} 91 / 676$ du 12 décembre 1991 (dite «directive nitrates »), devenue obligatoire durant l'automne sur les zones vulnérables. Les résultats de cette étude montrent que, globalement, les agriculteurs privilégient les couverts temporaires plurispécifiques durant les premières années en SDSC. Certaines associations peuvent effectivement s'avérer plus efficaces que des couverts mono-spécifiques pour répondre simultanément à plusieurs services. Couëdel et al. (2018) ont montré que l'association d'une légumineuse et d'une crucifère fournit un service de recyclage de l'azote aussi important qu'un couvert mono-spécifique de crucifère, en plus du service de fixation atmosphérique de l'azote par la légumineuse. Toutefois les couvertures végétales avec une espèce unique peuvent s'avérer plus efficaces pour différents objectifs que des associations d'espèces (Finney et al., 2016). La mise en place de couverts permanents, nécessitant une technicité plus importante, est appliquée par $18 \%$ des agriculteurs. Les repousses de cultures et les résidus tiennent une place non négligeable dans la couverture du sol (23 et $24 \%$ ).

À l'échelle de l'exploitation, l'adoption progressive du SDSC semble permettre aux agriculteurs de diminuer les risques en testant cette approche sur plusieurs parcelles sans que cela ne conduise à une adoption du SDSC sur l'ensemble de l'exploitation.

\section{Conclusion}

Cette étude avait pour objectif de faire le point sur les choix agronomiques réalisés par les agriculteurs lors de l'adoption du SDSC. Bien que l'adoption puisse être fortement influencée par les conditions locales (pédoclimatiques, économiques ou techniques), mais également par le réseau d'échange de chaque agriculteur, cette étude a permis de mettre en avant certaines similarités dans les choix agronomiques réalisés. Identifier ces similarités pourra, pour d'éventuelles adoptions futures du SDSC, constituer une base de la réflexion pour les agriculteurs.

La transition vers le SDSC se fait majoritairement de manière progressive. Certains choix apparaissent également partagés par la majorité des agriculteurs (utilisation de couverts végétaux plurispécifiques, ajout de légumineuses dans la rotation). Ces choix agronomiques résultent d'une volonté de la part des agriculteurs de diminuer les risques encourus lors de l'adoption et de partager de manière importante les connaissances. En replaçant le sol au centre de leur raisonnement et en maximisant les services attendus par une gestion agroécologique de ce dernier, ces agriculteurs proposent une autre approche de l'agriculture. $\mathrm{Au} \mathrm{vu} \mathrm{du}$ contexte politique global, le principal inconvénient du SDSC réside dans l'utilisation, durant l'interculture, de glyphosate, molécule au devenir encore incertain en France. En effet, $65 \%$ des agriculteurs enquêtés déclarent que le retrait du glyphosate se traduirait pour eux par un arrêt du SDSC.

\section{Matériel supplémentaire}

Données supplémentaires. Questions utilisées dans cette étude.

Le matériel supplémentaire est disponible sur http://www. cahiersagricultures.fr/10.1051/cagri/2020003/olm.

Remerciements. Cette enquête a été possible grâce aux financements du programme de recherche et d'innovation Horizon 2020 de l'Union européenne au titre de la convention de subvention $\mathrm{n}^{\mathrm{o}} 727321$ (IWMPraise) et de la convention de soutien à une allocation de thèse $n^{\circ}$ 2017-9201AAO048S01305 de la région Bourgogne-Franche-Comté. Nous remercions, pour leur participation à la diffusion de l'enquête, l'ensemble des réseaux d'agriculteurs (APAD, BASE, GIEE Du Sol Eau Soleil, AOC Sols, Club AgroEcos, GIEE MAGELLAN, Clé de Sol), les partenaires du projet (Terres Inovia, Arvalis), les représentants des chambres d'agriculture et d'organismes de conseil et le site A2C. Les agriculteurs sont remerciés pour leur participation. 


\section{Références}

Baeumer K, Bakermans W. 1974. Zero tillage. Advances in Agronomy 25(1): 77-123. DOI: 10.1016/S0065-2113(08)60779-8.

Barzman M, Bàrberi P, Birch A, Boonekamp P, Dachbrodt-Saaydeh S, Graf B, et al. 2015. Eight principles of integrated pest management. Agronomy for Sustainable Development 35(4): 1199-1215. DOI: 10.1007/s13593-015-0327-9.

Bijttebier J, Ruysschaert G, Hijbeek, R, Werner M, Pronk A, Zavattaro L, et al. 2018. Adoption of non-inversion tillage across Europe: Use of a behavioural approach in understanding decision making of farmers. Land Use Policy 78: 460-471. DOI: 10.1016/j. landusepol.2018.05.044.

Cameira M, Fernando R, Pereira L. 2003. Soil macropore dynamics affected by tillage and irrigation for a silty loam alluvial soil in southern Portugal. Soil and Tillage Research 70: 131-140. DOI: 10.1016/S0167-1987(02)00154-X.

Carof M, De Tourdonnet S, Coquet Y, Hallaire V, Roger-Estrade J. 2007. Hydraulic conductivity and porosity under conventional and no-tillage and the effect of three species of cover crop in northern France. Soil Use Management 23(3): 230-237. DOI: 10.1111/ j.1475-2743.2007.00085.x.

Clarke J, Clark W, Hancock M. 1997. Strategies for the prevention of development of pesticide resistance in the UK- Lessons for and from the use of herbicides, fungicides and insecticides. Pesticide Sciences 51(3): 391-397. DOI: 10.1002/(SICI)1096-9063(199711) $51: 3<391::$ AID-PS654>3.0.CO;2-M.

Constantin J, Beaudoin N, Laurent F, Cohan J-P, Duyme F, Mary B. 2011. Cumulative effects of catch crops on nitrogen uptake, leaching and net mineralization. Plant and Soil 341(1-2): 137-154. DOI: 10.1007/s11104-010-0630-9.

Cordeau S, Wayman S, Reibel C, Strbik F, Chauvel B, Guillemin J-P. 2018. Effects of drought on weed emergence and growth vary with the seed burial depth and presence of a cover crop. Weed Biology Management 18(1): 12-25. DOI: 10.1111/wbm.12136.

Couëdel A, Alletto L, Tribouillois H, Justes E. 2018. Cover crop crucifer-legume mixtures provide effective nitrate catch crop and nitrogen green manure ecosystem services. Agriculture, Ecosystems \& Environment 254: 50-59. DOI: $10.1016 / \mathrm{j}$. agee.2017.11.017.

Derpsch R, Friedrich T, Kassam A, Hongwen L. 2010. Current status of adoption of no-till farming in the world and some of its main benefits. International Journal of Agricultural and Biological Engineering 3 (1): 1-25. DOI: 10.3965/j.issn.1934-6344.2010.01.001-025.

Dorado J, López-Fando C. 2006. The effect of tillage system and use of a paraplow on weed flora in a semiarid soil from central Spain. Weed Research 46(5): 424-431. DOI: 10.1111/ j.1365-3180.2006.00526.x.

Finney D, White C, Kaye J. 2016. Biomass production and carbon/ nitrogen ratio influence ecosystem services from cover crop mixtures. Agronomy Journal 108(1): 39-52. DOI: 10.2134/ agronj15.0182.

House G, Parmelee R. 1985. Comparison of soil arthropods and earthworms from conventional and no-tillage agroecosystems. Soil and Tillage Research 5(4): 351-360. DOI: 10.1016/S0167-1987 (85)80003-9.

Laloy E, Bielders C. 2010. Effect of intercropping period management on runoff and erosion in a maize cropping system. Journal of Environmental Quality 39(3): 1001-1008. DOI: $10.2134 /$ jeq2009.0239.

Laurent F. 2015. L'Agriculture de Conservation et sa diffusion en France et dans le monde. Cybergeo: European Journal of Geography [11/10/2019]. http://journals.openedition.org/cyber geo/27284.

Meynard J-M, Messéan A, Charlier A, Charrier F, Fares M, Le Bail M, et al. 2013. Freins et leviers à la diversification des cultures. Etude au niveau des exploitations agricoles et des filières. Synthèse du rapport d'étude, INRA, $52 \mathrm{p}$.

Nielsen D, Lyon D, Higgins R, Hergert G, Holman J, Vigil M. 2016. Cover crop effect on subsequent wheat yield in the central Great Plains. Agronomy Journal 108(1): 243-256. DOI: 10.2134/ agronj2015.0372.

Osipitan O, Dille J, Assefa Y, Radicetti E, Ayeni A, Knezevic S. 2019. Impact of cover crop management on level of weed suppression: A meta-analysis. Crop Science 59(3): 833-842. DOI: 10.2135/ cropsci2018.09.0589.

Parr M, Grossman J, Reberg-Horton S, Brinton C, Crozier C. 2011. Nitrogen delivery from legume cover crops in no-till organic corn production. Agronomy Journal 103(6): 1578-1590. DOI: 10.2134/ agronj2011.0007.

Peigné J, Casagrande M, Payet V, David C, Sans F, Blanco-Moreno $\mathrm{JM}$, et al. 2016. How organic farmers practice conservation agriculture in Europe. Renewable Agriculture and Food Systems 31 (1): 72-85. DOI: $10.1017 / \mathrm{S} 1742170514000477$.

Reicosky DC. 2015. Conservation tillage is not conservation agriculture. Journal of Soil and Water Conservation 70(5): 103108. DOI: 10.2489 /jswc.70.5.103A.

Šarauskis E, Buragiene S, Masilionyte L, Romaneckas K, Avižienyte D, Sakalauskas A. 2014. Energy balance, costs and $\mathrm{CO}_{2}$ analysis of tillage technologies in maize cultivation. Energy 69: 227-235. DOI: 10.1016/j.energy.2014.02.090.

Schipanski M, Smith R, Gareau T, Jabbour R, Lewis D, Barbercheck $\mathrm{M}$, et al. 2014. Multivariate relationships influencing crop yields during the transition to organic management. Agriculture, Ecosystems \& Environment 189(1): 119-126. DOI: 10.1016/j. agee.2014.03.037.

Schwen A, Bodner G, Scholl P, Buchan G, Loiskandl W. 2011. Temporal dynamics of soil hydraulic properties and the waterconducting porosity under different tillage. Soil and Tillage Research 113(2): 89-98. DOI: 10.1016/j.still.2011.02.005.

Citation de l'article : Derrouch D, Dessaint F, Felten E, Chauvel B. 2020. L'adoption du semis direct sous couvert végétal : transition douce ou rupture? Cah. Agric. 29: 5. 